\title{
Oscillatory magnetic flux tube slippage in the plasma sheet
}

\author{
A. A. Petrukovich ${ }^{1}$, T.L. Zhang ${ }^{2}$, W. Baumjohann ${ }^{2}$, R. Nakamura ${ }^{2}$, A. Runov ${ }^{2}$, A. Balogh ${ }^{3}$, and C. Carr ${ }^{3}$ \\ ${ }^{1}$ Space Research Institute, 84/32 Profsoyuznaya st., Moscow, 117997, Russia \\ ${ }^{2}$ Space Research Institute, Austrian Academy of Sciences, Schmiedlstr. 6, 8042 Graz, Austria \\ ${ }^{3}$ The Blackett Laboratory, Imperial College London SW7 2BW, UK
}

Received: 27 December 2005 - Revised: 3 April 2006 - Accepted: 7 April 2006 - Published: 3 July 2006

\begin{abstract}
Cluster observations in the magnetotail revealed an abundance of strongly inclined current sheets. We determine the magnetic configuration of a particular subset of such phenomena: a series of sheet crossings, having significantly differing inclinations and occurring during quiet conditions. These wave-like variations appear to propagate azimuthally and their magnetic amplitude and magnetic gradient (current density) inside the sheet are proportional to their steepness (degree of inlcination). In spite of significant normal direction changes between neighboring crossings up to $150^{\circ}$, the magnetic field direction inside the neutral sheet remains almost constant. The wavelengths and spatial amplitudes are of the order of $2-5 R_{E}$. These observations are interpreted as crossings of a quasi-periodic dynamical structure produced by almost vertical slippage motion of the neighboring magnetic flux tubes in the high- $\beta$ plasma sheet, rather than large-scale flapping of a stationary structure.
\end{abstract}

Keywords. Magnetospheric physics (Current systems; Magnetotail; Plasma sheet)

\section{Introduction}

The multi-spacecraft Cluster project provides an opportunity to determine the gradient and orientation of a magnetic or plasma structure. The first four years of Cluster magnetotail observations revealed structural complexity of the plasma sheet with an abundance of crossings with significantly inclined current sheets (Sergeev et al., 2004; Runov et al., 2005a). In several targeted investigations, some such events were interpreted as a wavy displacement of the main crosstail current sheet plane, propagating flankward (Zhang et al., 2002), or as a quasi-stationary structure of vertically shifted flux tubes, flapping azimuthally around the spacecraft location (Petrukovich et al., 2003).

Correspondence to: A. A. Petrukovich

(apetruko@iki.rssi.ru)
Here we concentrate on a rather common type of observation: a series of current sheet crossings, in which nearby crossings (in time) have significantly differing or sometimes alternating inclination. This phenomenon can be understood (in a first approximation) as a wave of vertical displacement of a notional neutral sheet surface (e.g. Zhang et al., 2002, 2005). It is distinctly different from, for example, a back-and-forth flapping motion of a stationary configuration, which reveals itself as a series of current sheet crossings with the same inclination. The possibility and variants of such sheet modifications were earlier discussed by Lui et al. (1978); Lui (1984); McComas et al. (1986).

Since the main cross-tail current sheet is actually a 3-D object, formed by curved magnetic flux tubes, two variants of deformation can occur (Fig. 1). During a bend-type change flux tubes rotate, following the change in the sheet normal direction. Alternatively, during a slip-type (shear-type) change, flux tubes just shift (vertically) relative to their neighbors and the magnetic field direction inside a sheet is not changing. Additionally, in the course of bending, the current sheet thickness remains constant, while under a slip-type deformation the current sheet thickness diminishes proportionally to the cosine of the sheet tilt angle.

Taking advantage of multi-spacecraft Cluster and Double Star TC-1 observations we can distinguish these two variants of dynamical behavior and determine several characteristics of the observed oscillations. In the following sections the event selection is described, several typical events are presented and possible implications for local and large-scale plasma sheet structure are discussed.

\section{Event selection and the approach}

For this study we selected events with a "slow wave-like change" of $B_{x}$ (i.e. with at least two crossings in a row), occurring in a quiet high- $\beta$ plasma sheet with plasma bulk velocity below $100 \mathrm{~km} / \mathrm{s}$. Additionally it was required that current sheet properties (normal, velocity, etc.) are decipherable by the Cluster tetrahedron (Petrukovich et al., 2005), with

Published by Copernicus GmbH on behalf of the European Geosciences Union. 


\section{$\underbrace{\text { ZGSM }}_{\text {YGM }}$ \\ Nominal horizontal configuration}

Slip-type deformation: Flux-tubes shift, magnetic direction is constant, thickness decreases.
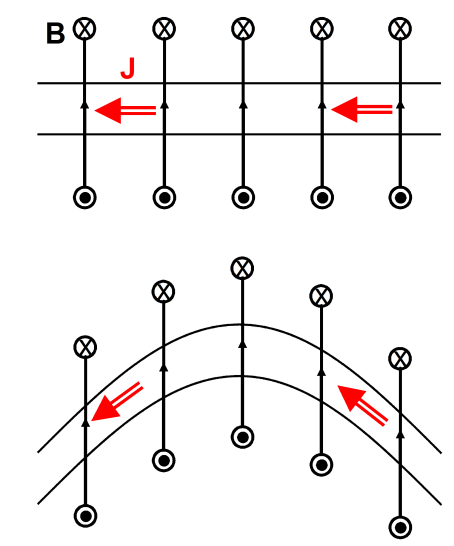

Bend-type deformation: Flux-tubes rotate, magnetic direction follows the normal, thickness is constant.

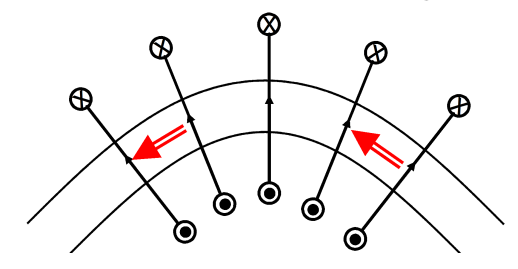

Fig. 1. Variants of the cross-tail current sheet deformation. The view in $Y Z$ GSM plane is shown, assuming nominal configuration with $B_{y}=0$. See text for details.

leading and trailing crossings in each oscillation exhibiting a significant difference in orientation, and both sheets moving in the same direction (actually always away from the tail center). However, contrary to the Runov et al. (2005a) data set, there were no additional limitations on the amplitude of the magnetic field changes. Finally, after visual scanning of 2001, 2002, and 2004 observations we selected several clear events (14.5 wave periods or 29 sheet crossings), detected on both magnetotail flanks.

Cluster and Double Star TC-1 4-s resolution magnetic field data (Balogh et al., 2001; Carr et al., 2005) were used for the analysis. Components $x, y, z$ are in the GSM frame of reference. The angles $\phi, \theta$ are in a special coordinate system, with $X$ as the polar axis (Petrukovich et al., 2005). Zero values of polar and azimuthal angles for a sheet normal correspond to a horizontal sheet, i.e. a normal along the $Z$ GSM direction. The polar angle $\theta$, the colatitude, is measured from the $Y Z$ plane. Positive values correspond to normals, inclined earthward, negative - tailward. The azimuthal angle $\phi$ (in the $Y Z$ plane) is measured from the $Z$ axis (positive for a normal with a positive $Y$ component).

With four-point observations one can determine the spatial gradient, assuming a constant gradient (linearity) on the scale of spacecraft separation, the stationarity of the configuration, and a constant uniform relative plasma frame velocity. Local (independent at each spacecraft) variations overlaying the large-scale change in question are neglected. In the magnetotail, plasma sheet observations of the magnetic gradient are usually interpreted in the approximation of a uniform planar current sheet crossing. The sheet's normal direction can then be assigned to the $B_{x}$ gradient direction (assuming that the actual magnetic maximum variance direction most likely is not orthogonal to $X$ ). This "magnetic normal" can be computed instantaneously for each set of magnetic field measurements by the four spacecraft. The alternative method is to determine the normal and velocity along the normal, analyzing interspacecraft time delays within the crossing (equivalent to the computation of the "time" gradient $d t / d r$ ) (Runov et al., 2005b). Magnetic gradient normals always point northward, while timing normals are in the direction of motion. Other independent sheet characteristics are the maximum variance direction defining the orientation of the main sheet magnetic component $B_{l}$, and the electric current direction (computed as $\nabla \times B)$.

For the majority of our events the timing and magnetic gradient normals were coincident and orthogonal to maximal variance and current vectors with an accuracy of about $10^{\circ}-15^{\circ}$. Therefore, the approximation of a planar sheet is acceptable. Since angles between the experimentally determined normal, maximal variance and shear directions are not exactly $90^{\circ}$, we established for each crossing a similar orthogonal proper frame of reference with $l$ along the maximal variance, $m=n_{b} \times l\left(n_{b}\right.$ is magnetic normal, averaged over the middle of the crossing, as described in the end of this section), $n=l \times m$. Note that while the final $l m n$ system is established only for the whole crossing, the magnetic normal can be computed with the time resolution of magnetic field vector measurements.

In the planar uniform current sheet approximation only the $B_{l}$ component is created by the cross-tail current and vanishes in the neutral sheet, while the rest of the magnetic field $\left(B_{m}, B_{n}\right)$ is constant and remains in the neutral sheet, reflecting the flux tube configuration, IMF influence, etc. Hereafter, the magnetic field $B_{n}, B_{m}$ (also converted back to GSM as $B_{x}^{\prime}, B_{y}^{\prime}, B_{z}^{\prime}$ ) will be called the "sheet magnetic field". In examining its changes from crossing to crossing, one can decide on the mode of sheet deformation, as explained in the Introduction and Fig. 1.

Finally, we describe an azimuthally propagating wave of the neutral sheet plane with a simple model: $\mathcal{Z}=\mathcal{Z}_{0} \cdot f((\omega-$ $\left.\left.k V_{y d}\right) t-k \mathcal{Y}\right)+V_{z d} t$, where $V_{z d}$ and $V_{y d}$ are Doppler velocities due to some background bulk motion; $\omega$ - wave frequency; $k$ - wave vector; $\mathcal{Z}, \mathcal{Y}$ - local vertical and azimuthal directions; $t$-time; $f(x)$ - some harmonic or other periodic function. Note that $\partial \mathcal{Z} / \partial \mathcal{Y}=\tan (\phi)=-\mathcal{Z}_{0} k f^{\prime}$, that is, the wavelength can be determined, if the sheet inclination and the oscillation amplitude are known. However, frequency $\omega$ remains unknown.

Table 1 contains spacecraft coordinates, solar wind conditions and neutral sheet model characteristics. Table 2 contains sheet characteristics: normal and maximal variance directions, sheet velocity along the normal (positive in $+Z$ direction), as well as the sheet magnetic field with subtracted $B_{l}$ maximal variance component. Table 3 contains wave parameters: angles between the actual sheet normal 
Table 1. General characteristics of the crossings: Date, GSM coordinates, IMF, solar wind (SW) dynamic pressure $P_{d}$, model neutral sheet (NS) location $Z$ and normal direction angles $\phi, \theta$.

\begin{tabular}{lllrrrr}
\hline Date & & \multicolumn{1}{l}{ Cluster-3 position, $R_{E}$} & $\mathrm{IMF} B_{y}, B_{z}, \mathrm{nT}$ & $\mathrm{SW} P_{d}$ & $\mathrm{NS} \mathrm{Z}, R_{E}$ & $\mathrm{NS} \phi, \theta$ \\
\hline 021024 & $03: 20-03: 40$ & $-12.2,12.3,-0.4$ & $0,-5$ & 2.1 & -0.9 & $-30.9,-16.0$ \\
021031 & $05: 20-05: 45$ & $-10.5,13.1,-1.0$ & $5,-4$ & 3.7 & 1.52 & $-45.4,-14.4$ \\
010803 & $08: 53-09: 25$ & $-16.9,-8.6,3.0$ & $-8,1$ & 6.0 & 1.99 & $-5.9,9.9$ \\
040803 & $06: 35-06: 50$ & $-15.9,-10.0,1.8$ & $-4,0$ & 0.6 & 1.52 & $-1.5,6.8$ \\
040803 & $06: 49-06: 59$ & $-15.9,-10.0,1.8$ & $-4,0$ & 0.6 & 1.57 & $-1.8,7.1$ \\
040803 & $06: 58-07: 08$ & $-16.0,-10.0,1.7$ & $-4,0$ & 0.6 & 1.62 & $-1.9,7.3$ \\
040803 & $07: 07-07: 16$ & $-16.0,-10.0,1.7$ & $-4,0$ & 0.6 & 1.65 & $-2.1,7.4$ \\
040803 & $07: 20-07: 30$ & $-16.1,-10.0,1.6$ & $-4,1$ & 0.6 & 1.85 & $-2.4,8.2$ \\
040803 & $07: 28-07: 43$ & $-16.1,-10.0,1.6$ & $-4,1$ & 0.6 & 1.92 & $-2.5,8.5$ \\
040803 & $08: 00-08: 20$ & $-16.2,-10.0,1.4$ & $-4,2$ & 0.6 & 2.05 & $-2.7,9.1$ \\
040803 & $08: 17-08: 30$ & $-16.2,-10.0,1.3$ & $-4,2$ & 0.6 & 2.12 & $-3.0,9.5$ \\
040803 & $08: 30-09: 00$ & $-16.3,-10.0,-1.2$ & $-4,3$ & 0.6 & 2.14 & $-4.5,9.7$ \\
040803 & $09: 07-09: 22$ & $-16.3,-10.0,1.0$ & $-2,4$ & 0.6 & 2.17 & $-6.0,10.0$ \\
\hline
\end{tabular}

Table 2. The sheet configuration: the angles of normal and maximum variance directions, velocity along the normal (signed), sheet magnetic field as $B_{m}, B_{n}$ and converted to GSM $B_{x}^{\prime}, B_{y}^{\prime}, B_{z}^{\prime}$, the angle between normals and sheet magnetic fields in the neighboring crossings $\alpha_{n n}$, $\alpha_{b b}$.

\begin{tabular}{|c|c|c|c|c|c|c|c|c|}
\hline Date & & Normal $\theta, \phi$ & $\operatorname{Max} \operatorname{var} \theta, \phi$ & $V_{n}$ & $B_{m}, B_{n}$ & $B_{x}^{\prime}, B_{y}^{\prime}, B_{z}^{\prime}$ & $\alpha_{n n}$ & $\alpha_{b b}$ \\
\hline 021024 & $03: 20-03: 40$ & $\begin{array}{r}10 .,-9 . \\
-30 .,-73 .\end{array}$ & $\begin{array}{r}50.8,-103.2 \\
56.9,-92.2\end{array}$ & $\begin{array}{r}30.1 \\
-37.9\end{array}$ & $\begin{array}{r}-1.2,8.0 \\
9.0,4.4\end{array}$ & $\begin{array}{r}-0.17,-2.00,7.8 \\
-0.33,-0.90,10.0\end{array}$ & 73. & 9. \\
\hline 021031 & $05: 20-05: 45$ & $\begin{array}{r}16 ., 5 . \\
-38 .,-61 .\end{array}$ & $\begin{array}{l}44.4,-95.9 \\
48.2,-98.1\end{array}$ & $\begin{array}{r}22.6 \\
-25.2\end{array}$ & $\begin{array}{r}-2.5,3.7 \\
2.9,2.6\end{array}$ & $\begin{array}{r}-1.1,-1.50,4.0 \\
-0.37,-0.95,3.8\end{array}$ & 82. & 11. \\
\hline 010803 & $08: 53-09: 25$ & $\begin{array}{r}-9 ., 54 . \\
15 .,-63 . \\
-4 ., 58 .\end{array}$ & $\begin{array}{l}66.0,131.5 \\
70.7,128.3 \\
65.6,125.3\end{array}$ & $\begin{array}{r}-15.2 \\
8.6 \\
-39.2\end{array}$ & $\begin{array}{r}-12.1,4.2 \\
12.1,5.1 \\
-12.9,3.3\end{array}$ & $\begin{array}{r}4.7,-4.40,11.0 \\
2.4,1.40,12.8 \\
4.3,-2.90,12.8\end{array}$ & $\begin{array}{l}118 . \\
121 .\end{array}$ & $\begin{array}{l}29 . \\
19 .\end{array}$ \\
\hline 040803 & $06: 35-06: 50$ & $\begin{array}{l}-26 ., 46 . \\
39 .,-84 .\end{array}$ & $\begin{array}{l}54.3,97.3 \\
54.3,94.8\end{array}$ & $\begin{array}{r}-35.1 \\
15.2\end{array}$ & $\begin{array}{l}-5.4,2.2 \\
6.1,1.87\end{array}$ & $\begin{array}{r}1.5,-1.40,5.5 \\
0.78,-0.57,6.3\end{array}$ & 136. & 11. \\
\hline 040803 & 06:49-06:59 & $\begin{array}{l}-21 ., 45 . \\
33 .,-27 .\end{array}$ & $\begin{array}{l}56.7,105.9 \\
56.6,100.1\end{array}$ & $\begin{array}{r}-28.9 \\
28.4\end{array}$ & $\begin{array}{r}-4.7,2.8 \\
2.3,4.2\end{array}$ & $\begin{array}{l}1.91,-1.60,4.9 \\
0.64,-0.14,4.7\end{array}$ & 87. & 20. \\
\hline 040803 & 06:58-07:08 & $\begin{array}{r}-14 ., 39 . \\
15 .,-9 .\end{array}$ & $\begin{array}{l}63.0,86.2 \\
56.9,79.3\end{array}$ & $\begin{array}{r}-29.5 \\
24.0\end{array}$ & $\begin{array}{l}-4.8,2.4 \\
-0.4,4.1\end{array}$ & $\begin{array}{l}0.86,-2.00,4.9 \\
0.32,-1.23,3.9\end{array}$ & 55. & 6. \\
\hline 040803 & 07:07-07:16 & $\begin{array}{r}-9 ., 18 . \\
11 .,-41 .\end{array}$ & $\begin{array}{r}66.0,64.6 \\
71.0,125.1\end{array}$ & $\begin{array}{r}-10.1 \\
30.0\end{array}$ & $\begin{array}{r}-2.5,2.2 \\
4.4,4.1\end{array}$ & $\begin{array}{l}0.13,0.13,2.9 \\
0.95,0.95,5.9\end{array}$ & 61. & 9. \\
\hline 040803 & $07: 20-07: 30$ & $\begin{array}{r}-8 ., 32 . \\
35 .,-40 .\end{array}$ & $\begin{array}{l}57.7,72.0 \\
51.2,99.8\end{array}$ & $\begin{array}{r}-24.2 \\
15.1\end{array}$ & $\begin{array}{r}-3.6,2.2 \\
3.9,4.1\end{array}$ & $\begin{array}{l}0.32,-1.78,3.9 \\
0.82,-0.07,5.6\end{array}$ & 80. & 24. \\
\hline 040803 & $07: 28-07: 43$ & $\begin{array}{l}-10 ., 30 . \\
18 .,-16 .\end{array}$ & $\begin{array}{l}54.2,87.6 \\
43.1,61.6\end{array}$ & $\begin{array}{r}-23.1 \\
13.5\end{array}$ & $\begin{array}{r}-4.2,3.3 \\
0.4,3.8\end{array}$ & $\begin{array}{r}1.12,-1.76,4.9 \\
-0.12,-1.69,3.4\end{array}$ & 53. & 15. \\
\hline 040803 & 08:00-08:20 & $\begin{array}{l}38 .,-55 . \\
-26 ., 57 .\end{array}$ & $\begin{array}{r}55.7,98.8 \\
55.2,101.4\end{array}$ & $\begin{array}{r}26.6 \\
-14.0\end{array}$ & $\begin{array}{r}5.1,3.4 \\
-5.8,2.4\end{array}$ & $\begin{array}{l}0.40,0.33,6.1 \\
1.5,-1.04,6.0\end{array}$ & 122. & 16. \\
\hline 040803 & 08:17-08:30 & $\begin{array}{l}34 .,-67 . \\
-28 ., 50 .\end{array}$ & $\begin{array}{l}57.4,95.8 \\
57.7,98.7\end{array}$ & $\begin{array}{r}27.6 \\
-19.4\end{array}$ & $\begin{array}{r}5.0,2.9 \\
-5.8,2.5\end{array}$ & $\begin{array}{l}0.37,-0.00,5.8 \\
1.31,-1.17,6.1\end{array}$ & 126. & 13. \\
\hline 040803 & 08:30-09:00 & $\begin{array}{l}23 .,-31 . \\
-24 ., 42 .\end{array}$ & $\begin{array}{r}50.9,119.8 \\
55.9,86.4\end{array}$ & $\begin{array}{r}14.9 \\
-16.8\end{array}$ & $\begin{array}{r}1.9,3.3 \\
-6.2,2.2\end{array}$ & $\begin{array}{r}1.24,0.30,3.6 \\
1.35,-2.39,6.0\end{array}$ & 85. & 26. \\
\hline 040803 & 09:07-09:22 & $\begin{array}{l}27 .,-91 . \\
-34 ., 72 .\end{array}$ & $\begin{array}{l}62.5,99.2 \\
59.5,94.3\end{array}$ & $\begin{array}{r}-6.2 \\
-24.6\end{array}$ & $\begin{array}{r}7.2,0.6 \\
-7.3,0.9\end{array}$ & $\begin{array}{l}0.80,-0.39,7.2 \\
0.92,-1.00,7.2\end{array}$ & 163. & 4. \\
\hline
\end{tabular}


Table 3. Calculated sheet parameters: angle between the sheet normal and the normal to the model neutral sheet $\alpha_{n n s}$, the range of magnetic oscillation $\Delta B_{l}$, gradient along the normal $\partial B_{l} / \partial n$, estimates of wavelength $\lambda$ and period.

\begin{tabular}{|c|c|c|c|c|c|c|}
\hline \multicolumn{2}{|l|}{ Date } & \multirow{3}{*}{$\begin{array}{l}\alpha_{n n s} \\
-33.8 \\
-40.9\end{array}$} & \multirow{3}{*}{$\begin{array}{r}\Delta B_{l}, \mathrm{nT} \\
23.6 \\
38.1\end{array}$} & \multirow{3}{*}{$\begin{array}{r}\partial B_{l} / \partial n, \mathrm{nT} / R_{E} \\
24.6 \\
36.8\end{array}$} & \multirow{3}{*}{$\begin{array}{r}\lambda, R_{E} \\
5.4 \\
4.9\end{array}$} & \multirow{3}{*}{$\begin{array}{r}\text { Half-period, } \mathrm{s} \\
450 \\
450\end{array}$} \\
\hline 021024 & $03: 20-03: 40$ & & & & & \\
\hline & & & & & & \\
\hline \multirow{2}{*}{\multicolumn{2}{|c|}{021031 05:20-05:45 }} & 58.3 & 41.8 & 33.6 & 4.5 & 660 \\
\hline & & -27.3 & 26.0 & 29.3 & 6.0 & 360 \\
\hline \multirow[t]{3}{*}{010803} & $08: 53-09: 25$ & 62.5 & 29.0 & 35.0 & 2.9 & 660 \\
\hline & & -55.8 & 23.0 & 21.7 & 4.0 & 660 \\
\hline & & 65.1 & 31.1 & 27.7 & 3.9 & 360 \\
\hline \multirow[t]{2}{*}{040803} & $06: 35-06: 50$ & 56.5 & 18.3 & 21.9 & 3.1 & 360 \\
\hline & & -79.9 & 20.8 & 20.9 & 3.1 & 540 \\
\hline \multirow[t]{2}{*}{040803} & $06: 49-06: 59$ & 53.8 & 13.2 & 18.6 & 2.7 & 270 \\
\hline & & -34.8 & 10.0 & 11.6 & 4.7 & 270 \\
\hline \multirow[t]{2}{*}{040803} & 06:58-07:08 & 45.8 & 9.2 & 15.1 & 2.6 & 240 \\
\hline & & -10.3 & 6.6 & 9.7 & 11.8 & 240 \\
\hline \multirow[t]{2}{*}{040803} & 07:07-07:16 & 25.8 & 7.5 & 14.3 & 3.7 & 330 \\
\hline & & -38.5 & 7.5 & 11.2 & 3.3 & 210 \\
\hline \multirow[t]{2}{*}{040803} & $07: 20-07: 30$ & 37.9 & 6.1 & 11.2 & 3.9 & 255 \\
\hline & & -43.5 & 6.7 & 10.0 & 5.6 & 255 \\
\hline \multirow[t]{2}{*}{040803} & $07: 28-07: 43$ & 37.2 & 5.4 & 13.1 & 2.1 & 210 \\
\hline & & -16.1 & 6.0 & 7.1 & 12.5 & 330 \\
\hline \multirow[t]{2}{*}{040803} & 08:00-08:20 & -55.0 & 16.8 & 14.5 & 4.8 & 390 \\
\hline & & 67.7 & 14.7 & 23.1 & 1.1 & 390 \\
\hline \multirow[t]{2}{*}{040803} & $08: 17-08: 30$ & -63.2 & 11.4 & 13.9 & 2.9 & 360 \\
\hline & & 63.4 & 12.7 & 20.3 & 2.1 & 360 \\
\hline \multirow[t]{2}{*}{040803} & 08:30-09:00 & -28.6 & 7.9 & 8.2 & 6.3 & 630 \\
\hline & & 56.5 & 15.2 & 18.1 & 3.1 & 630 \\
\hline \multirow{2}{*}{\multicolumn{2}{|c|}{040803 09:07-09:22 }} & -81.0 & 21.6 & 37.2 & 1.7 & 450 \\
\hline & & 85.8 & 25.7 & 55.2 & 1.4 & 270 \\
\hline
\end{tabular}

and the normal to model neutral sheet, defining local vertical; $\Delta B_{l}-$ magnetic field oscillation amplitude (computed as the difference between maximal and minimal $B_{l}$ ), $\partial B_{l} / \partial n$ - magnetic field gradient across the sheet, wavelength, crossing duration, equaled to the wave half-period. Field, as well as gradient values and normal angles in Tables 2, 3 are averaged over the "middle" of each crossing, defined as $B_{l}^{a}-2 \mathrm{nT}<\mathrm{B}_{1}<\mathrm{B}_{1}^{\mathrm{a}}+2 \mathrm{nT}$, where $B_{l}^{a}=\left(B_{l}^{\min }+B_{l}^{\max }\right) / 2$.

\section{Events}

\subsection{October 2002}

Figure 2 presents a typical single wave, registered at the far dusk flank. A positive-negative-positive signature in the $B_{x}$ magnetic component (Fig. 2a), if measured with a single spacecraft, would be interpreted as an up-down-up, cross-tail current sheet motion. However, the four-point analysis (calculation of the instant $B_{x}$ gradient, equivalent to the sheet normal, as explained in Sect. 2) reveals significant sheet tilts, changing in the course of the event from $\phi \sim 5^{\circ}$ to $\phi \sim-61^{\circ}$ (Fig. 2g). Figure 3 suggests reconstruction of this event, probably taking into account the rather the inclined background configuration (the normal to the model neutral sheet (Tsyganenko and Fairfield, 2004) has $\phi \sim-45^{\circ}$ ).

Comparing two crossings, the $B_{m}$ and $B_{n}$ (Figs. 2e, f) components change significantly, while $B_{z}$ (which is almost orthogonal to $B_{l}$ ) is not changing. Note that two different lmn frames are actually used in Figs. 2d, e, and f for the first and second crossings.

Table 2 contains values of sheet magnetic field $B_{m}, B_{n}$ and $B_{x}^{\prime}, B_{y}^{\prime}, B_{z}^{\prime}\left(B_{m}, B_{n}\right.$, transformed back to the $x y z$ frame). The difference between the normal directions of two neighboring crossings is $82^{\circ}$, while the sheet magnetic field orientation changes only $11^{\circ}$ (two last columns of Table 2 , see also Fig. 3). The two $B_{l}$ directions are close and almost earthward in consistency, with the expected flux tube plane orientation at a such location. The wavelength was found to be of the order of a couple of Earth radii (Table 3). 


\subsection{August 2001}

This event at the dawn flank was described in detail earlier by Zhang et al. (2002) and includes at least three clear sheet crossings (Fig. 4). The model sheet is almost horizontal here (Table 1). The main features of these crossings (stability of $B_{l}$ and sheet magnetic field directions, etc.) are similar to that of the previous example (Tables 2,3). A new and interesting feature is sharp rectangular normal direction changes at waves maxima/minima, more typical to a triangular wave.

\subsection{August 2004}

Figure 5 presents probably the most fortuitous observation of the phenomenon, providing the possibility to study a wave train with a variety of amplitudes and tilts under rather stable external conditions. Cluster was located at $(-16.0,-10.0$, 1.5) $\mathrm{R}_{E}$, the normal to the model neutral sheet (Tsyganenko and Fairfield, 2004) was just $9^{\circ}$ from the GSM vertical, and the IMF had an azimuthal orientation (Table 1). We analyzed waves, marked by the dark green rectangles ( 20 crossings in 10 "pairs", Fig. 5a). It is interesting to note, that even some small maxima of $B_{x}$ of the order of $5 \mathrm{nT}$ between 07:00 and 08:00 UT, exhibit an alternating sheet orientation at their leading and trailing edges (Figs. 5d, e). Crossings parameters are detailed in Tables 1-3.

In Fig. 6a the difference between the normal directions for pairs of consecutive crossings was compared with the difference between the respective sheet's magnetic field directions. The changes in sheet normal direction were $50-150^{\circ}$, while the magnetic orientation was rather stable, varying only 5$25^{\circ}$. According to Table 2, $l$ axis directions for all crossings are similar and are pointing to the Earth.

There is a tendency for the total sheet magnetic field (mostly $B_{z}$ ) to be smaller for smaller tilts (Fig. 6b). In agreement with Fig. 6a, the angle between the normals and the sheet magnetic field directions increases for more tilted events, so that the guide field component $\left(B_{m}\right)$ dominates in more vertical sheets (Fig. 6c).

In the slip deformation model, the $B_{l}$ magnetic gradient in the flux tube plane is constant, while the gradient component along the normal should increase proportionally to an inverse cosine of the effective sheet tilt angle. In the bend deformation model the gradient along the normal is constant. Changes in $d B_{l} / d n$ are more consistent with the slip variant (Fig. 7b). An unexpected feature is the clear proportionality between the magnetic amplitudes and the tilt angles (relative to the normal to the model neutral sheet) of the waves, so that larger waves are steeper (Fig. 7a). Finally, there is some relation between wavelengths $\left(2-5 \mathrm{R}_{E}\right)$ and tilt angles (Fig. 7c), but there is no clear frequency dependence (not shown here). Determination of a wavelength depends on a type of $f(x)$ function (see Sect. 2), defining the wave form. We used the harmonic wave profile.

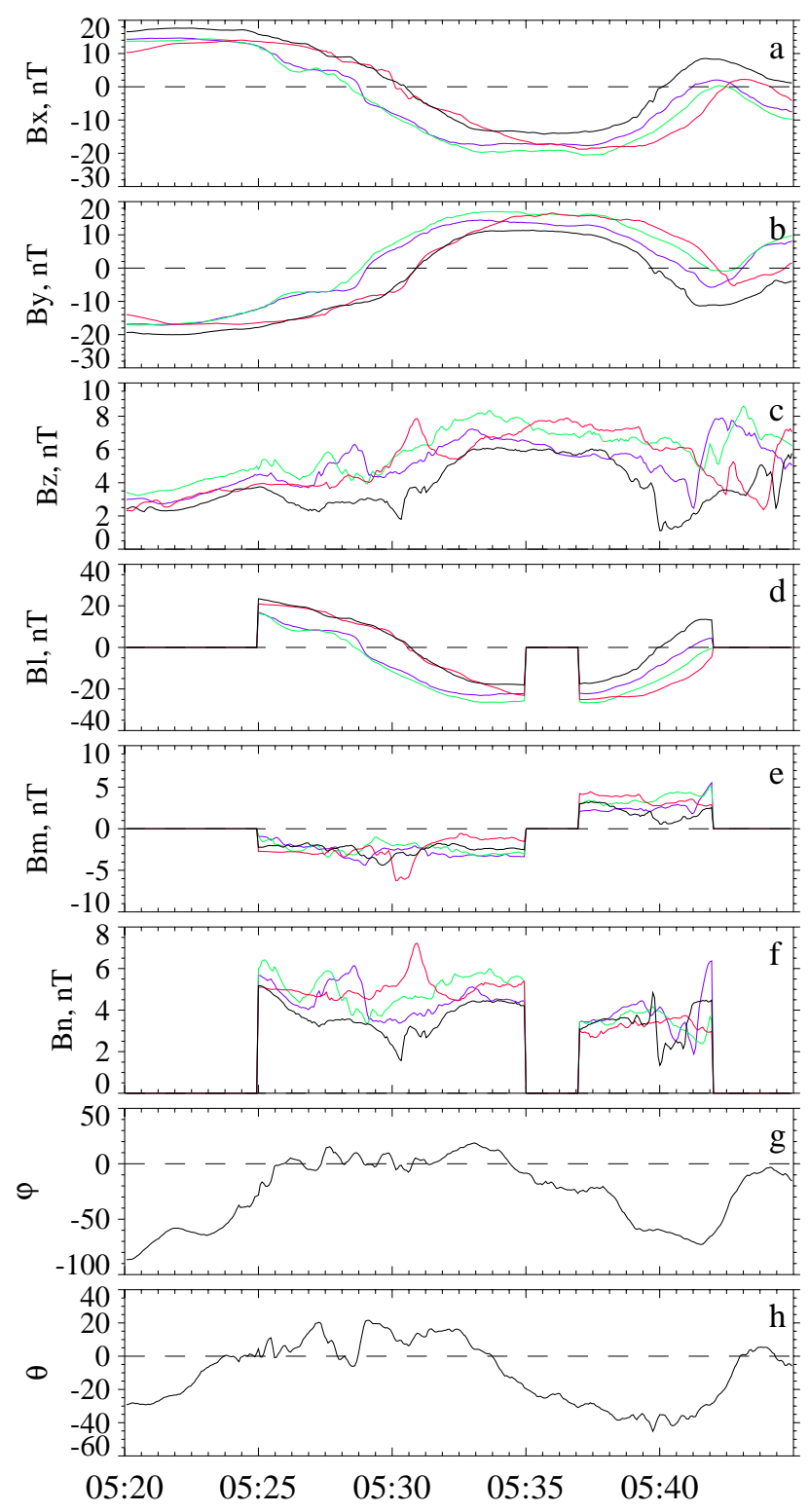

Fig. 2. Event 31, October 2002: Magnetic field in GSM, Imn frames of reference (note that $l m n$ frames are different at the first and second crossings, therefore data are in two pieces). In ( $\mathrm{g}, \mathrm{h})$ panels normal direction in GSM. C1,C2,C3,C4 spacecraft are denoted by standard colors - black, red, green, blue, respectively.

The Double Star TC-1 satellite (at $-10.66,-6.97,2.89 R_{E}$ GSM at 08:00 UT), which is usually at the same local time, but closer to Earth than Cluster, helps to check the radial extent of these oscillations (Fig. 8). Though TC-1 was in the outer plasma sheet $\left(B_{x} \sim 30 \mathrm{nT}\right)$ and amplitudes of magnetic variations are smaller, some correlation with the larger Cluster oscillations is evident. 


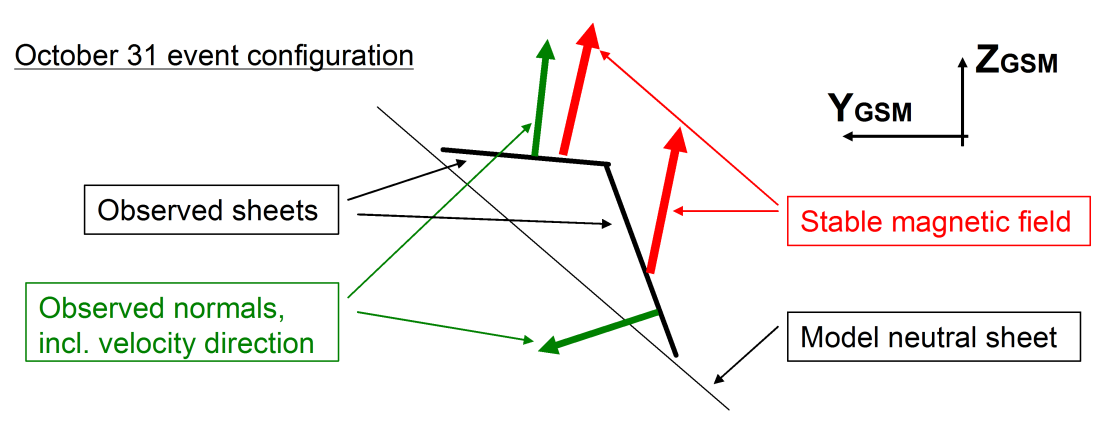

Fig. 3. The sketch of the 31 October 2002 double crossing. Shown are the tilted model neutral sheet plane, as well as crossing with significant planes and normals (incl. direction of propagation along the normal) of two crossings, sheet magnetic field direction.

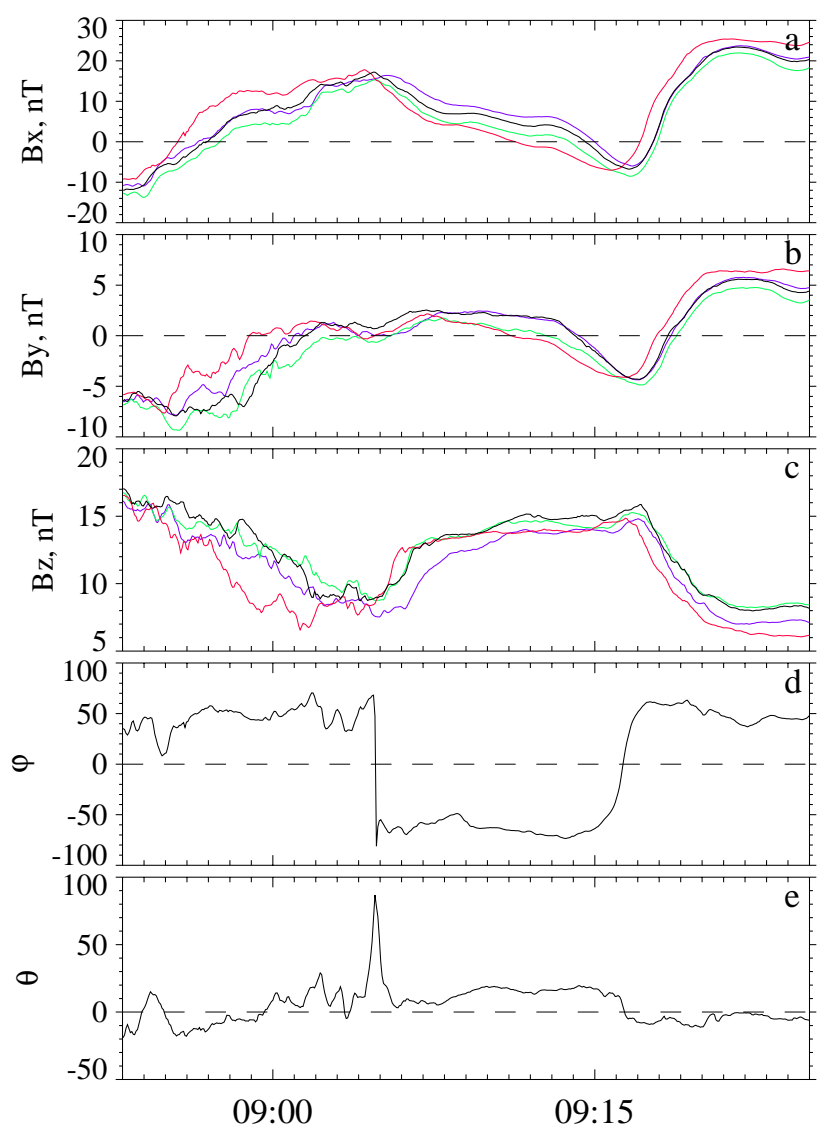

Fig. 4. Event 3 August 2001: Magnetic field and normal direction angles in GSM frame of reference. Colors are as in Fig. 2.

\section{Summary of observations}

We analyzed a special sort of repeating quiet current sheet crossings with significant orientation change between neighboring events. These variations have the following common features:

1. The magnetic field direction (magnetic field with subtracted $B_{l}$ ) inside neighboring current sheets is almost the same in the geophysical frame of reference and changes significantly in the $\operatorname{lm} n$ frame. The change is especially noticeable in $B_{m}$, which often reverses its sign from crossing to crossing. The maximal variance direction is also stable in consecutive crossings and points approximately earthward.

2. The magnetic gradient along the normal (current density) increases with the increase of the tilt.

3. Magnetic amplitudes are related to tilt angles: larger waves are steeper. Wavelengths are $2-5 R_{E}$.

4. Oscillations have some extent along the tail, being observed on several spacecraft.

\section{Discussion}

Our observational findings (1) and (2) definitely support a model of an azimuthally propagating slip-type displacement of magnetic flux tubes. All selected events happened to be on the flanks, and are characterized by small $B_{y}$ and large $B_{z}$ magnetic components, suggesting rather thick plasma sheets. On a completely speculative basis, bending deformation might be more probable for thin intense current sheets with large $B_{y}$ and small $B_{z}$, when neighboring flux tubes are more coupled.

Earlier, a similar technique was suggested by Lui (1984), to distinguish between different types of plasma sheet deformations. It was based on an analysis of magnetic field rotations, observed by a single spacecraft in the course of a sheet crossing. For example, in one case a hump-like configuration was revealed (Lui et al., 1978).

The accuracy of the estimates of spatial scales (amplitudes and wavelengths) critically depends on the quality of gradient measurements. The four-point gradient estimation assumes a linearity of magnetic profiles (constant gradient). For a traversal of the inner part of a Harris profile, the gradient would be underestimated by a factor of 0.8-0.9 (Runov et al., 2005a). Wavelength estimates can also differ by a factor of 1.5, depending on a choice of waveform (harmonic or 


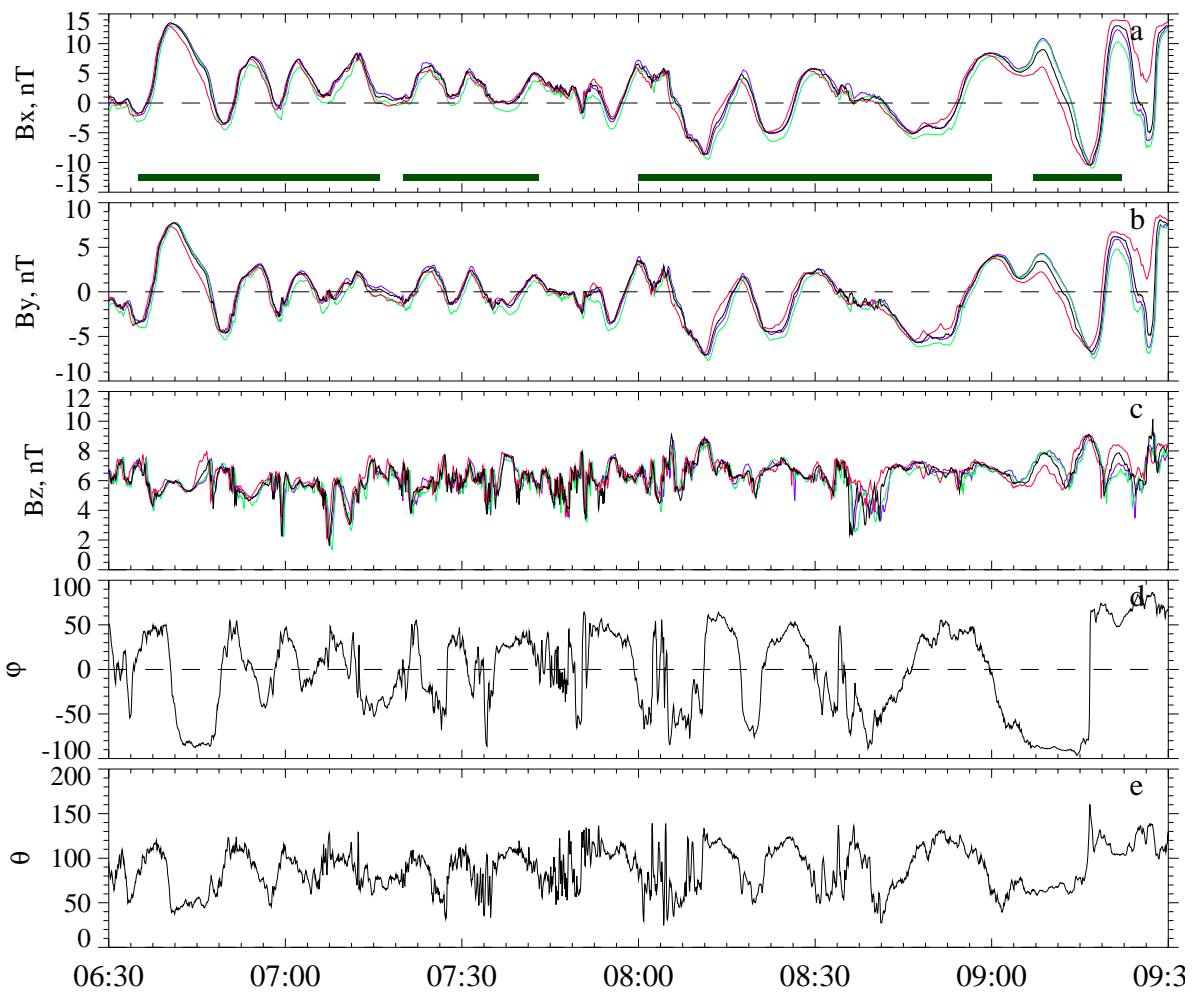

Fig. 5. Event 3 August 2004: Magnetic field and normal direction angles in GSM frame of reference. Colors are as in Fig. 2. Dark green rectangles at the bottom of panel (a) denote intervals under study.

triangular). Periods and velocities of variations under study (minutes and tens of $\mathrm{km} / \mathrm{s}$ ) are of the order of that of tail flapping, therefore background conditions may change significantly in the course of an event and significantly affect the detected wave characteristics.

If one considers such a series of sheet crossings as some proper wave mode of the plasma sheet, then the typical spatial amplitude and wavelength are of the order of several $R_{E}$. It should be noted, however, that only leading and trailing edges of an assored wave are actually observed as two sheet crossings (see also discussion by Runov et al. (2005a)). The whole wave profile is unknown and is not necessarily sinusoidal (event, 3 August 2001). An interesting feature is a link between the tilt angle of a sheet and the magnetic amplitude of a variation, making larger-amplitude waves steeper. Unfortunately it was detected only in one extremely fortuitous event of 3 August 2004 and cannot be verified on larger statistics because of significant spreads in data, taken from completely different observations.

The observed wavy deformation of the plasma sheet has the mesoscale scope in the vertical and azimuthal directions, extending a few Earth radii. Considering the radial direction, this deformation can be alternatively understood as an azimuthally and radially localized dynamic "hump", or as a coherent motion of a "slice" of plasma sheet flux tubes, occupying a significant range of downtail distances. This problem cannot be solved with Cluster spacecraft data only. Double Star TC-1 spacecraft, being about $6 R_{E}$ closer to Earth, revealed some current sheet motion (seen as $B_{x}, B_{y}$ variations), consistent with the Cluster variations and indicating a possibly significant radial extent of the deformation. However, it is unclear whether the smaller amplitude at the TC-1 position was due to its more distant location from the neutral sheet, or due to a smaller amplitude change closer to Earth. In a number of other observations, comparable amplitude magnetic field variations or simultaneous current sheet crossings were detected by spacecraft 6 and $10, R_{E}$ apart radially and aligned in local time (Petrukovich et al., 2003; Zhang et al., 2005). However, for those events, full identification of the deformation mode was not performed.

The discussed phenomenon should be understood as a dynamic modification of the inner (high- $\beta$ ) plasma sheet - a formation of an intensified layer with varying tilt, embedded in a much thicker plasma sheet, rather than a steady sheet profile with some large-scale bulk tail motion. Figure 9 is the sketch of the modification in a plane orthogonal to the $B_{l}$ direction, $B_{y}=0$. Up and down motions of slipping flux tubes are seen as variations in contours of equal $B_{l}$ (marked levels $\pm B_{L}, \pm B_{0}, 0$ ). It is assumed that the oscillation is smaller far from the neutral sheet: the amplitude of the variation in contours $\pm B_{L}$ is smaller than in contours $\pm B_{0}$. 

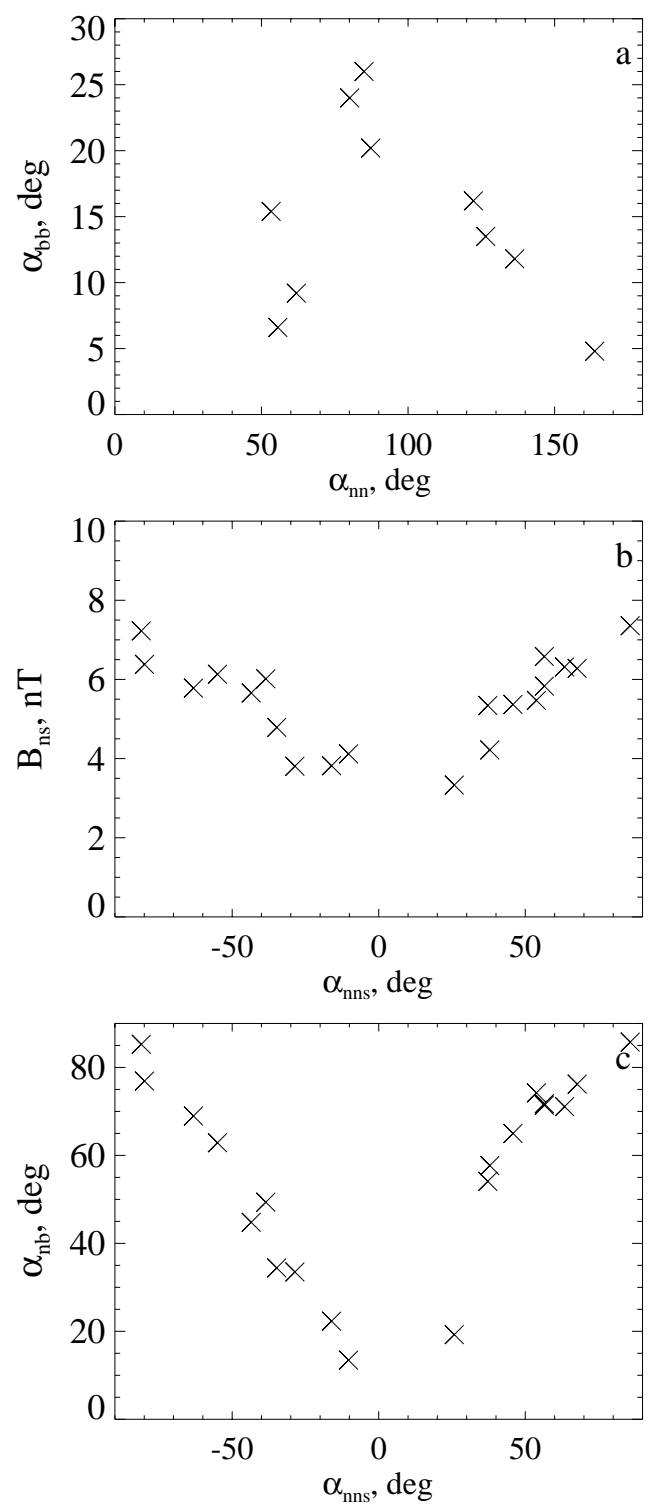

Fig. 6. Properties of current sheet crossings for 3 August 2004: (a) The angles between sheet's magnetic field directions of neighboring crossings compared with the difference of the normal directions. (b) Total sheet magnetic field $\left(\sqrt{B_{n}^{2}+B_{m}^{2}}\right)$ versus the angle between the sheet normal and the normal to the model neutral sheet for each crossing. (c) The same for the angle between sheet magnetic field and normal.

The magnetic amplitude of a wave is equal to the maximal $B$ level, crossing the nominal neutral sheet plane in the course of an oscillation ( $B_{0}$ in the middle of the picture). If a virtual spacecraft is located near the nominal neutral sheet plane, it will observe magnetic oscillations $\sim \pm B_{0}$, interpreted as crossings of inclined current sheets. As a consequence of slip deformation, the distance between the $\pm B_{0}$ levels (thickness) is smaller and the current density is larger, than that for the horizontal static configuration, and
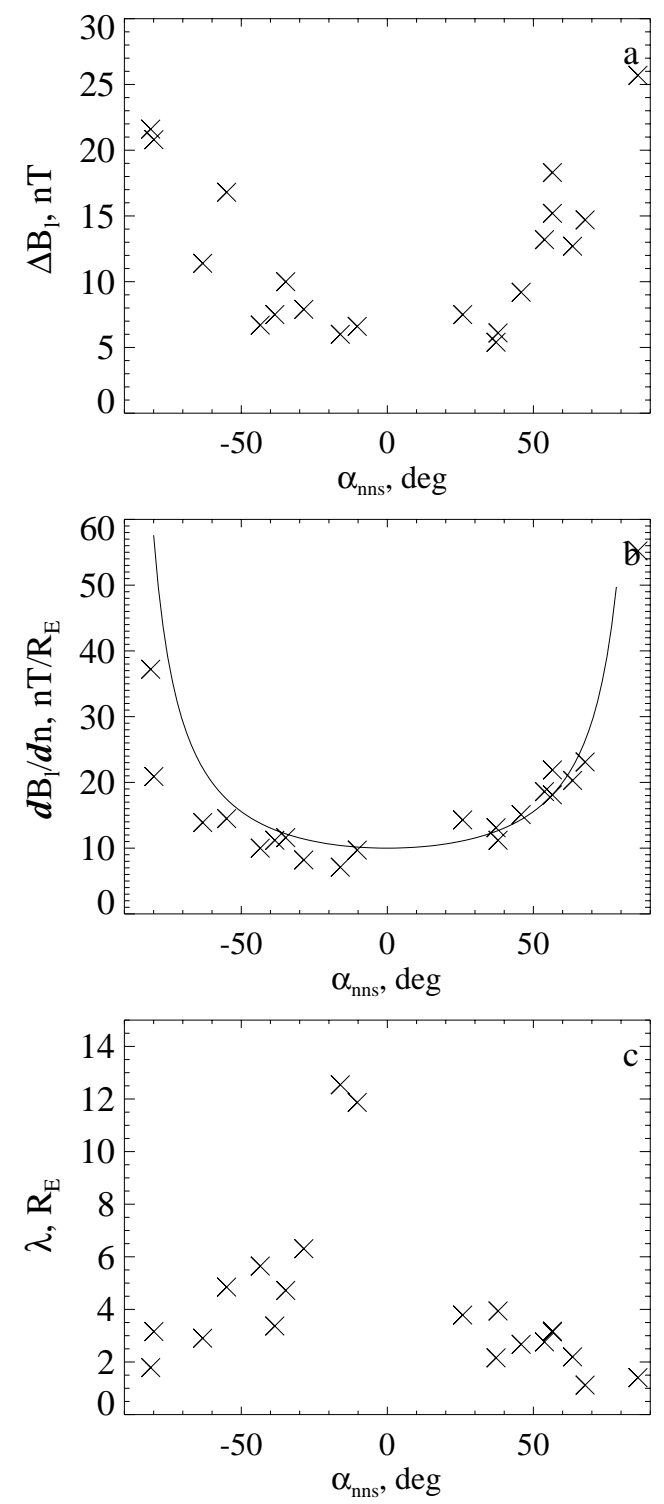

Fig. 7. Properties of current sheet crossings for 3 August 2004: (a) Magnetic amplitudes of crossings versus the angle between the sheet normal and the normal to the model neutral sheet for each crossing. (b) The same for the $B_{l}$ gradient along the normal. Solid line denotes the inverse cosine dependence. (c) The same for wavelengths.

the thickness is inversely proportional to the tilt. Therefore, the dynamic inner part might be interpreted as the intensified (relative to Harris profile) inner current layer, embedded in a thick current sheet $\left(J_{L}\right)$, supporting the large-scale magnetic field reversal $\pm B_{L}$. This dynamic layer has no permanent thickness, since it depends on the amplitude of oscillation and tilt.

A recently suggested type of ballooning mode, describing a displacement of the magnetic flux tubes in the $X Z$ plane from the equilibrium position in the antisymmetric 


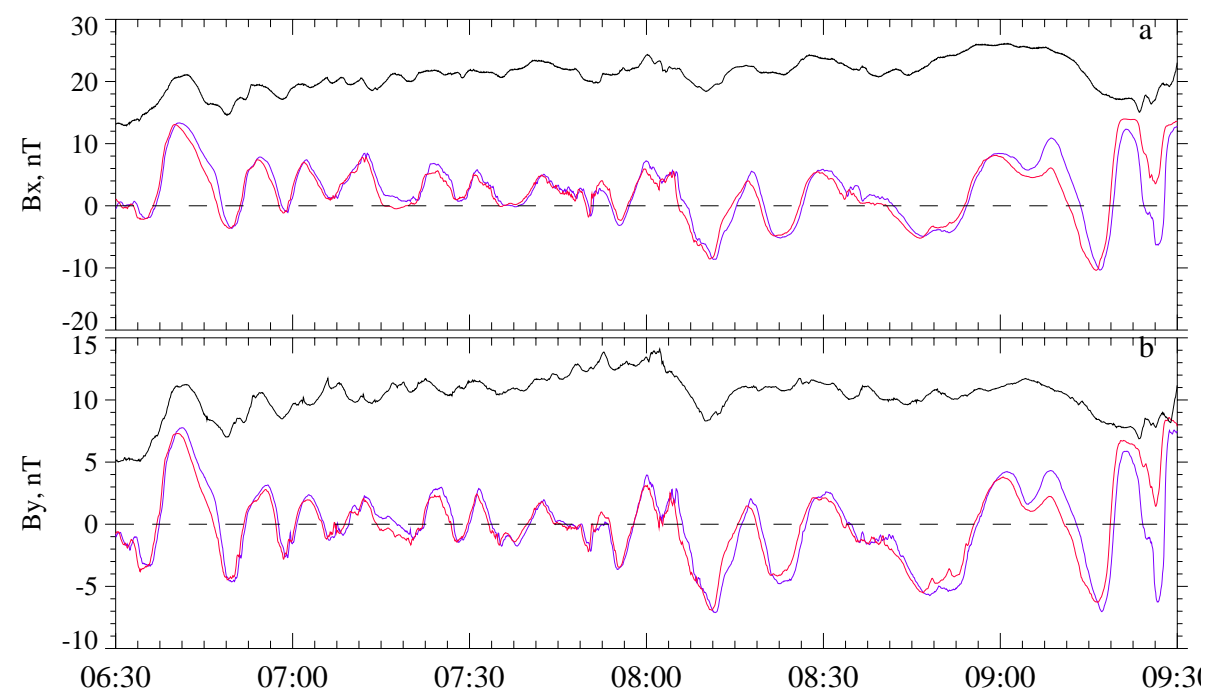

Fig. 8. 3 August 2004, GSM magnetic field for Cluster-2 (red), Cluster-4 (blue), Double Star-1 (black).

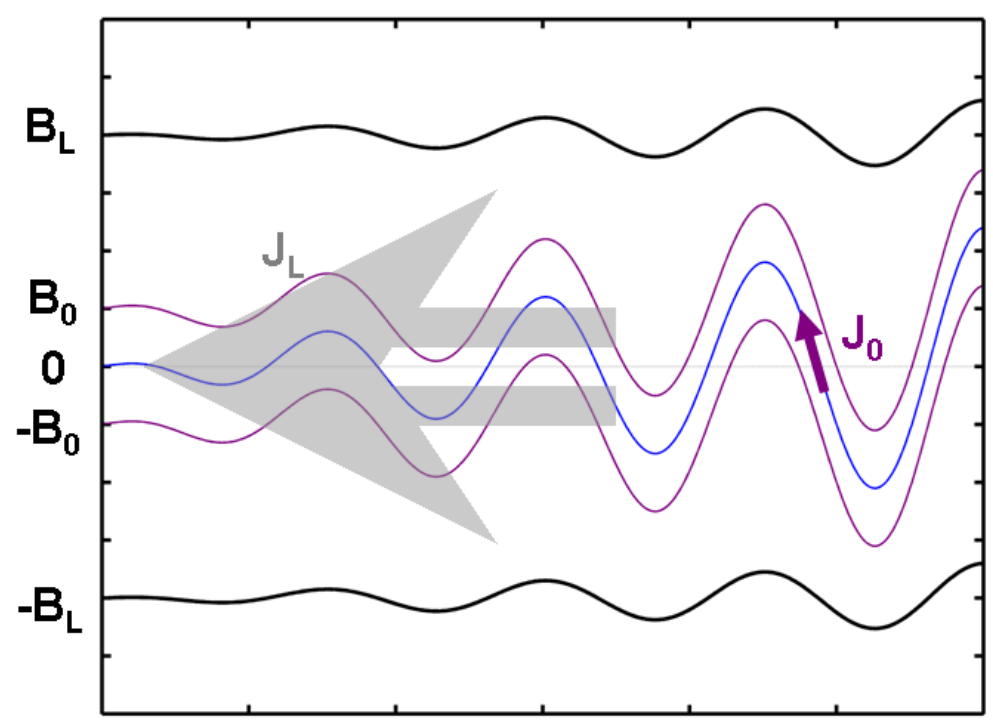

Fig. 9. The scheme of dynamic sheet modification. See text for details. The thin variable current layer $J_{0}$ creates the magnetic wave $\pm B_{0}$, observed by a spacecraft as a series of sheet crossings. The full magnetic gradient $\pm B_{L}$ is supported by a much thicker horizontal current $J_{L}$.

standing wave, seen as a kink-like deformation of the current sheet (Golovchanskaya and Maltsev, 2005), fits well our observations. A further investigation is necessary to understand whether the oscillation amplitude and wavelength (or sheet tilt) are coupled in this mode, as was observed in the experiment.

\section{Conclusions}

Our investigation targeted wave-like variations in a rather quiet, thick (large $B_{z}$ ) sheet. However, similar fast crossings of strongly inclined sheets are frequently observed in rather diverse conditions. Accurate determination of the magnetic configuration, which is needed to decide on the type of sheet deformation, is not always possible, especially in the case of isolated single crossings, or in highly disturbed conditions. Further insight into the Cluster magnetotail data, including 
new analysis methods, is necessary to solve this problem, on the whole. Besides a proper understanding of the plasma sheet structure, the investigation may reveal details that are of interest for basic plasma physics, namely, self-consistent adaptation of the current density to varying sheet thickness and values of normal and guide magnetic components.

Acknowledgements. The work was supported in part by the INTAS Grant 03-51-3738 and Russian grant MD-3036.2006.5. A. A. Petrukovich would like to acknowledge academic exchange program and hospitality of IWF.

Topical Editor I. A. Daglis thanks D. H. Fairfield and V. Angelopoulos for their help in evaluating this paper.

\section{References}

Angelopoulos, V., Baumjohann, W., Kennel, C. F., Coroniti, F. V., Kivelson, M. G., Pellat, R., Walker, R. J., Lühr, H., and Paschmann, G.: Bursty bulk flows in the inner central plasma sheet, J. Geophys. Res., 97, 4027-4039, 1992.

Balogh, A., Carr, C. M., Acuna, M. H., et al.: The Cluster magnetic field investigation: overview of in-flight performance and initial results, Ann. Geophys., 19, 1207-1217, 2001.

Carr, C., Brown, P., Zhang, T. L. et al.: The Double Star magnetic field investigation: instrument design, performance and highlights of the first year's observations, Ann. Geophys., 25, 27132732, 2005.

Golovchanskaya, I. V. and Maltsev, Y. P.: On the identification of plasma sheet flapping waves observed by Cluster, Geophys. Res. Lett., 32, L02102, doi:10.1029/2004GL021552, 2005.

Lui, A. T. Y., Meng, C.-I., and Akasofu, S.-I.: Wavy nature of the magnetotail neutral sheet, Geophys. Res. Lett., 5, 279-282, 1978.

Lui, A. T. Y.: Characteristics of the cross-tail current in the Earth's magnetotail, in: Magnetospheric Currents, AGU Geophysical Monograph 28, 158-170, 1984.
McComas, D. J., Russell, C. T., Elphic, R. C., and Bame, S. J.: The near-Earth cross-tail current sheet: Detailed ISEE 1 and 2 case studies, J. Geophys. Res., 91, 4287-4301, 1986.

Petrukovich, A. A., Baumjohann, W., Nakamura, R., Balogh, A., Mukai, T., Glassmeier, K.-H., Rème, H., and Klecker, B.: Plasma sheet structure during strongly northward IMF, J. Geophys. Res., 108(A6), 1258, doi:10.1029/2002JA009738, 2003.

Petrukovich, A. A., Baumjohann, W., Nakamura, R., Runov, A., and Balogh, A.: Cluster vision of the magnetotail current sheet on a macro-scale, J. Geophys. Res., 110, A06204, doi:10.1029/2004JA010825, 2005.

Runov, A., Sergeev, V. A., Baumjohann, W., Nakamura, R., Apatenkov, S., Asano, Y., Volwerk, M., Vörös, Z., Zhang, T. L., Petrukovich, A., Balogh, A., Sauvaud, J.-A., Klecker, B., and Rème, H.: Electric current and magnetic geometry in flapping magnetotail current sheets, Ann. Geophys., 23, 1391-1403, 2005 a.

Runov, A., Sergeev, V. A., Nakamura, R., Baumjohann, W., Zhang, T. L., Asano, Y., Volwerk, M., Vörös, Z., Balogh, A., Sauvaud, J.-A., and RÈme, H.: Reconstruction of the magnetotail current sheet structure using multi-point Cluster measurements, Planetary and Space Science, 53, 237-243, 2005 b.

Sergeev, V., Runov, A., Baumjohann, w., Nakamura, r., Zhang, T. L., Balogh, A., Louarn, P., Sauvaud, J.-A., and Rème, H.: Orientation and propagation of current sheet oscillations, Geophys. Res. Lett., 31, L05807, doi:10.1029/2003GL019346, 2004.

Tsyganenko, N. A. and Fairfield, D. H.: Global shape of the magnetotail current sheet as derived from Geotail and Polar data, J. Geophys. Res., 109, A03218, doi:10.1029/2003JA010062, 2004.

Zhang, T. L., Baumjohann, W., Nakamura, R., Balogh, A., and Glassmeier, K.-H.: A wavy twisted neutral sheet observed by Cluster, Geophys. Res. Lett., 29, 1899, doi:10.1029/2002/GL015544, 2002.

Zhang, T. L., Nakamura, R., Volwerk, M., Runov, A., Baumjohann, W., Eichelberger, H. U., Carr, C., Balogh, A., Sergeev, V., Shi, J. K., and Fornacon, K.-H.: Double Star/Cluster observation of neutral sheet oscillations on 5 August 2004, Ann. Geophys., 23, 2909-2914, 2005. 